\title{
El operador no en el español de Centroamérica: análisis tipológico ${ }^{1}$
}

\section{Gisselle Herrera Morera²}

Universidad Nacional, Costa Rica

\begin{abstract}
resumen
El estudio analiza el operador no en el español de Centroamérica desde una perspectiva tipológica y representa un aporte a los estudios de descripción formal del español en la región. La muestra proviene de la base de datos del Programa de Lingüística Hispánica (PROLHISPA) de la Universidad Nacional, Costa Rica.
\end{abstract}

\section{abstract}

This study analyzes the operator no in Central American Spanish from a typological perspective and represents a contribution to research on the formal description of Spanish in the region. The sample is taken from the database of the Hispanic Linguistics Program (PROLHISPA), of the Universidad Nacional, Costa Rica.

Palabras clave: negación, operador no, español en Centroamérica, análisis a base de corpus

Keywords: negation, operator no, Central American Spanish, corpus-based analysis

1 Versión revisada de la ponencia leída en el I Simposio Internacional del Programa de Lingüística Centroamericana (PROLINCA) «Centroamérica: un microcosmo lingüístico», llevado a cabo los 7 y 8 de mayo 2012, en la ciudad de Heredia, en el campus Omar Dengo de la Universidad Nacional de Costa Rica. Recibido: 6 de diciembre de 2011; aceptado: 6 de marzo de 2012.

2 Escuela de Literatura y Ciencias del Lenguaje. Correo electrónico: giselle.herreramorera@ gmail.com

$$
L_{\text {etras }} 51 \text { (2012), ISSN 1409-424X }
$$




\section{Introducción}

El este estudio se analiza la negación operada mediante la partícula no en el español de Centroamérica desde una perspectiva tipológica. No cubre toda la variedad de formas que en español se utilizan para la negación. Esta categoría constituye un universal del lenguaje que interesa a expertos de varias disciplinas y su camino recorrido es bastante antiguo; remonta a Aristóteles y llega hasta nuestros días con innumerables publicaciones ${ }^{3}$ Bosque $^{4}$, Lámina ${ }^{5}$, Horn ${ }^{6}$, van der Wouder ${ }^{7}$, Sánchez ${ }^{8}$, Horn y Kato ${ }^{9}, \mathrm{Kasim}^{10}$, entre otras). Las tipologías lingüísticas son significativas por su efecto en el análisis de la estructura de una lengua, pues la mayoría de las tipologías, sobre todo las tradicionales, suele mezclar enfoques o disciplinas. Si bien hay algunos trabajos acerca del español en Centroamérica (Quesa$\left.\mathrm{da}^{11}\right)$, no hay uno referente a la negación, basado en una tipología lingüística específica.

Para el análisis se empleó un corpus de la base de datos del Programa de Lingüística Hispánica (PROLHISPA) ${ }^{12}$ de la Universidad

3 La cantidad y variedad de las investigaciones no ha de extrañar ya que el tema ha sido objeto de estudio de la lógica, la psicolinguística y la semiótica, entre otras.

4 Ignacio Bosque, Sobre la negación (Madrid: Cátedra, 1980).

5 Xenia Lámina, «Sobre la asimetría de la forma y el contenido de las oraciones negativas en español», en Linguistique générale et linguistique romance. Histoire de la grammaire. Actes du XVII Congrès internacional de linguistique et philologie romanes (Aix-en-Provence, 29 agosto-3 setiembre), 1(1983): 243-248.

6 Laurence Horn, A Natural History of Negation. (Chicago: Chicago University Press, 1989).

7 Tom van der Wouden, Negative Contexts (Londres: Routledge, 1997).

8 Cristina Sánchez, «La negación», en Ignacio Bosque y Violeta Demonte (Dirs.), Gramática descriptiva de la lengua española 2 (Madrid: Espasa, 1999) 2561-2634.

9 Laurence Horn y Yasuhiko Kato (Eds.), Negation and Polarity: Syntactic and Semantic Perspectives (Oxford: Oxford University Press, 2000).

10 Sahar Kasim, Estudio comparativo de los procedimientos de la negación en español y en árabe. Tesis doctoral. (Granada: Editorial de la Universidad de Granada, 2009).

11 Miguel Ángel Quesada, «El español de América Central ayer, hoy y mañana», Boletín de Filología, XLIII (2008): 145-174. Miguel Ángel Quesada, «El español de América Central», Manuel Alvar (Dir.), Manual de dialectología hispánica. El español de América (Barcelona: Ariel,1996).

12 Los parámetros utilizados para la selección de los informantes fueron tres: haber nacido en la capital, tener un nivel de instrucción mínimo de primaria y pertenecer a uno de los siguientes tres grupos etarios: Grupo 1 (20-34), Grupo 2 (35-59) y Grupo 3 (60 en adelante). 
Nacional, el cual reúne veintisiete horas de grabación (un promedio de cuarenta y cinco minutos por informante) de entrevistas libres de treinta y seis hablantes centroamericanos (seis hombres y seis mujeres por cada capital centroamericana). La variedad elegida es la coloquial estándar (dialecto urbano) ${ }^{13}$.

La estructura de este artículo es la siguiente: después de esta introducción donde se explica los objetivos y estructura, en la segunda parte se esbozan las tipologías morfosintácticas existentes (Givón ${ }^{14}$ y Payne ${ }^{15}$ ) y se presenta lo referente a la negación y la partícula no en el español; en la tercera, se analizan los diferentes casos obtenidos de la base de datos de PROLHISPA; y finalmente se exponen las conclusiones.

\section{La negación como parámetro morfosintáctico}

\section{Tipología de la negación}

Payne ${ }^{16}$ y Givón ${ }^{17}$ distinguen tres tipos de negación: la léxica o inherente, la morfológica y la sintáctica o analítica. En esta investigación, interesa la negación sintáctica, basada en las relaciones gramaticales de los elementos que conforman una cláusula. Sin embargo, es conveniente describir algunos aspectos de la negación morfológica para tener una perspectiva global.

13 La decisión de limitar la procedencia de los hablantes a las capitales está en consonancia con el hecho de que en los países centroamericanos existe una estructura política y económica fuertemente centralizada, la cual ha llevado a la concentración de la mayoría de la población en las capitales (o en sus afueras); además, en ellas se halla un poder centrifugal que incorpora variedades de centro y periferia y las expande luego homogenizadoramente a las demás (p. ej.: vía medios de comunicación). Más información se puede encontrar en Giselle Herrera, Viviana Núñez y Juan Diego Quesada, «El español de Centroamérica: materiales para su estudio», Revista Voces, 1 (2012): 61-86.

14 Talmy Givón, Syntax. An Introduction (Vols. I y II) (Amsterdam: John Benjamins, 2001).

15 Thomas Payne, Describing Morphosyntax. A Guide for Field Linguistics (Cambridge: Cambridge University Press, 1997).

16 Payne, 282-284.

17 Givón, 395-396. 


\section{Morfología}

Givón ${ }^{18}$ plantea dos vías mediante las cuales emergen los marcadores de negación: a) verbos principalmente negativos que toman los complementos verbales bajo su alcance y b) intensificadores negativos que provienen de objetos dentro de la frase verbal. Explica que el primer grupo está conformado por verbos de modalidad negativa inherente, como fail, lack, refuse o decline (en inglés); así como el empleo de afijos, que ejemplifica con los siguientes ejemplos datos de la lengua bemba.

a. uku-bula

'to avoid', 'to lack' inf-avoid

b. uku-boomba

'to work' inf-work

c. n-a-bula uku-boomba

'I avoided working' I-past-avoid- inf-work

d. uku-bulaa-boomba inf-avoid-work

e. n-a-bulaa-boomba I-past-neG-word 'not to work'

'I didn't work', 'I failed to work'

El segundo grupo de marcas de negación son los intensificadores negativos enfáticos, que provienen del objeto transitivo. Inicialmente, tales intensificadores se agregan a la construcción negativa existente como énfasis o mayor especificación. Una vez establecidos, pueden dar origen a una doble negación no enfática. En tal construcción, estos intensificadores pierden su fuerza y se hacenclíticos;

18 Givón, 382. 
llegan incluso a remplazar el viejo marcador de negación. Como ejemplo, Givón ${ }^{19}$ señala a continuación lo ocurrido en el francés.
(2) a. je ne march pas
(pas 'step')
I neG march step
'I don't march'
(Hist.: 'I don't march even a step')
b. je ne connais personne (personne 'person') I neG know person
'I don't know anybody'
c. je ne sais rien
(*ren 'thing')
I neG know thing
'I don't know anything'

\section{Sintaxis}

Para Payne y Givón, las construcciones negativas suponen múltiples operadores, sean un afijo o una partícula, dos partículas, o una partícula o un afijo además de un cambio del orden de la palabra. Del mismo modo, la sintaxis de la negación tiene que ver con dos parámetros: la posición del marcador y el alcance. Tanto Givón ${ }^{20}$ como Payne ${ }^{21}$ consideran los anteriores aspectos y coinciden en que existen dos tipos de negación: la de la proposición completa, denominada negación de cláusulas o negación de fv y la negación de constituyentes o negación de fn. La negación de cláusula generalmente ocurre en el contexto de alguna presuposición y funciona negando u oponiéndose a la afirmación de lo presupuesto:

19 Givón, 268.

20 Givón, 382.

21 Payne, 282. 
Por ejemplo, si yo digo que Jorge no limpió la cocina, yo probablemente asuma que el destinatario presupone que Jorge limpió, o podría haber limpiado la cocina. A este respecto, las cláusulas negativas son similares funcionalmente a las cláusulas de foco contrastivo [...], y en consecuencia las cláusulas negativas y las de foco contrastivo son con frecuencia similares formalmente ${ }^{22}$.

Givón ${ }^{23}$ señala que cuando una presuposición — manifestada formalmente en términos de una cláusula - es negada, su valor de verdad lógica se invierte, de modo que siendo ase verada como verdadera ahora es falsa, pero el efecto de la negación sobre una proposición en una lengua natural es más complejo. Es más frecuente que una parte de una proposición negativa esté en el alcance de la negación, mientras el resto queda protegido. La porción de la cláusula protegida del alcance de la negación es su parte presupuesta. Givón ${ }^{24}$ agrega que la ne gación de cláusula o fv es el más común de los tipos de negación y tiene las siguientes características semánticas: a) tiende a negar solo la parte aseverada pero no la parte presupuesta de la afirmación correspondiente, b) tiende a excluir el sujeto del alcance de la negación, y c) es el tipo de negación menos específico, menos focalizado. No sorprende encontrar los morfemas que marcan negación fv como su operador, sean clíticos de fv, afijos oflexivos, pues dejan el sujeto fuera del alcance sintáctico. Para Givón, esta coincidencia de que el alcance sintáctico refleje el semántico está mediatizada por las dos vías diacrónicas que se mencionadas antes. Además de la típica exclusión del sujeto, la ne gación de fv puede usarse para limitar la porción de la cláusula que se encuentra bajo el alcance de la negación (neG-scope). Una forma de hacerlo es combinandolanegación de la fv y elénfasis contrastivoen los constituyentes de la cláusula para producir una negación focalizada (focused

22 Payne, 282.

23 Givón, 378.

24 Givón, 382. 
negation). En tales construcciones, el constituyente focalizado es el único que cae bajo el alcance de la negación y el resto de la cláusula es la presuposición.

Cuando los participantes opcionales, incluso los adverbios, se encuentran presentes en la cláusula, tienden a atraer el foco de la negación, lo cual deja el resto de la cláusula para ser presupuesto. Por ello, además de la negación focalizada (narrow scoped), la mayoría de las lenguas también tiene otros tipos de negación sintáctica que permiten que una marca negativa se una a un constituyente no-verbal de la cláusula (sujeto, objeto directo, objeto indirecto, predicado nominal o adverbio). A esta negación, Givón ${ }^{25}$ la denomina negación enfática o negación de FN. Payne ${ }^{26}$ aclara que este tipo de negación está asociado a constituyentes particulares de la cláusula, por ejemplo: I have no bananas; también, argumenta que su efecto puede ser similar o idéntico y que es menos común, como recurso gramatical, que la negación de cláusulas. Otro aspecto importante que menciona Givón $^{27}$ es que el constituyente negado en FN es no referencial.

En síntesis, Givón y Payne proponen dos tipos básicos de negación según el componente morfosintáctico modificado de una lengua: la negación morfológica y la negación sintáctica. A su vez, se puede encontrar otra subdivisión dependiente del ámbito afectado de la cláusula: negación de cláusula y negación de constituyentes; es decir, la negación de fiv, cuyo constituyente afectado dependerá del alcance de la negación, y la de FN, en la cual la fuerza recae en el constituyente al que se le haya agregado la marca u operador de negación: sujeto, objeto directo, objeto indirecto, predicado nominal o adverbio.

25 Givón, 392.

26 Payne, 282.

27 Givón. 


\section{La negación en español}

Sánchez ${ }^{28}$ define la negación como el conjunto de procedimientos gramaticales para llevar a cabo el acto de negar, es decir expresar la falsedad, inexactitud, irrealidad o no realización de un hecho, concepto o proposición; y agrega que el más habitual de estos procedimientos en español consiste en la anteposición del adverbio no al verbo; el resultado es una oración que declara la inadecuación entre sujeto y predicado o de la proposición entera con la realidad ${ }^{29}$. A ambos casos los llama negación oracional, aunque afirma que también existen en español palabras y sintagmas que poseen un significado negativo inherente, en virtud del cual solo pueden aparecer en oraciones de significado igualmente negativo. A esta concordancia de rasgos léxicos se la denomina polaridad negativa $(\mathrm{PN})$ y a los elementos sometidos a ella términos de polaridad negativa (TPN). Como ha quedado dicho, para esta investigación únicamente se tomarán como objeto de análisis las oraciones que emplean el operador no. Sánchez también menciona la negación de constituyentes, en la cual la negación afecta únicamente al sintagma al que precede, y aclara que en la mayoría de los casos corresponde a estructuras correctivas, en las cuales se niega un elemento para afirmar otro en su lugar, y que corresponde a una manifestación del foco de la negación; como ejemplos cita los siguientes:

28 Sánchez, 2563.

29 Según la disciplina o el enfoque adoptado, así serán las discrepancias entre los investigadores; por ejemplo, para Sánchez la anteposición del adverbio no al verbo da como resultado una oración que declara la inadecuación entre sujeto y predicado o de la proposición entera con la realidad. Por su parte, en su ponencia «La negación en español». ASELE. Actas VI. (Universidad de Valladolid, 1995), Beatriz Sanz Alonso aclara "Con esto queremos decir que negación no es lo contrario de afirmación; que muchas veces esta oposición ni siquiera existe; y que no es cierto lo que propugna la gramática tradicional de que el adverbio -o mejor dicho, el modulador noniega la relación entre sujeto y predicado. Primero, porque esta unión es permanente y segundo, porque este no puede tener varias y variadas funciones: entre otras, puede funcionar como oración en la réplica, por ejemplo, o lo podemos encontrar casi como un ilativo sin una función sintáctica determinada. Así, la relación sujeto-predicado será exactamente igual en la oración quiero que vengas que en no quiero que vengas, o que la que pueden tener ambos sintagmas en el nexus subordinado quiero que no vengas o no quiero que no vengas, porque el no sólo incide sobre el predicado" (379). 
(3) No por eso vamos a enfadarnos.

(4) No mucha gente sabe eso.

(5) María comió no peras, sino manzanas.

Negación y el orden de los constituyentes en español

El orden de los constituyentes en español es SVO no rígido y la frecuencia relativa del orden SV vs. VS está sujeta a diferentes variaciones dependientes del período histórico, del dialecto, el grado de formalidad, de licencias poéticas, entre otras ${ }^{30}$. El cuadro 1 muestra la frecuencia de distribución de los órdenes VS/SV y OV/ VO realizado por Givón ${ }^{31}$ en un texto contemporáneo en español, el cual tiende a confirmar la anterior aseveración.

\section{Cuadro 1. Distribución de frecuencia de los órdenes VS/SV y OV/VO en español contemporáneo, en Givón (2001)}

\begin{tabular}{|l|c|c|l|r|r|}
\hline Sujeto & $\mathbf{n}$ & $\boldsymbol{\%}$ & \multicolumn{1}{c|}{ Objeto } & n & \multicolumn{1}{c|}{$\%$} \\
\hline vS & 20 & 26,3 & ov & 2 & 2,6 \\
\hline Sv & 56 & 73,7 & vo & 75 & 97,4 \\
\hline Total: & 76 & 100,0 & Total: & 77 & 100,0 \\
\hline
\end{tabular}

Para Sánchez ${ }^{32}$, el marcador de negación en las cláusulas en español es preverbal. Entre el marcador de negación y el verbo hay elementos como el reflexivo, el objeto directo o el indirecto, e insiste en la importancia del orden en la negación, pues variar la posición del marcador no, que ella analiza como adverbio, a menudo conlleva agramaticalidad de la oración. Los resultados del estudio tienden a ratificar esa afirmación; sin embargo, como veremos más adelante, existen casos como (6) y (7) que rompen con lo establecido.

30 Bosque (1980) adopta el análisis de la oración como predicado oracional y la existencia de una estructura subyacente VSO, propuestas ambas que parten de la semántica generativa.

31 Givón, 276.

32 Sánchez, 2577. 
(6) Ajá, y los dulces típicos hay un montón, pero yo no/por ejemplo no como dulces típicos; no mucho me gustan (GT-H-1).

(7) ...a/en sí, así juegos no mucho les gusta; no, no les llama/así juegos comoo basquet o algo así nooo... deportes no les no les llama la atención (GT-H-2).

\section{Alcance de la negación en español}

En una cláusula negativa, generalmente se expresa que existe una situación, evento y estado de cosas opuesto a lo que se presupone. La negación es un operador sintáctico y, como todo operador, tiene un determinado alcance. Por consiguiente, en términos formales, si un operador se combina con una expresión $(\mathrm{O}, \mathrm{E}), \mathrm{E}$ está dentro del alcance de O. Para $\mathrm{Crystal}^{33}$, en español el alcance de la negación se acostumbra a extender desde la palabra negativa hasta el final de la cláusula; permite pues contrastes como Deliberadamente no se lo pregunté (= no se lo pregunté). No obstante, la negación puede afectar cualquier segmento lingüístico, sea a la oración, a la cláusula, al sintagma o a las palabras autosemánticas: sustantivos, adjetivos, pronombres, verbos, adverbios y las formas no personales del verbo. Las unidades que no admiten la negación en español son los determinantes: el artículo, los adjetivos demostrativos, los adjetivos posesivos antepuestos o apocopados; los pronombres átonos y clíticos en general; las conjunciones (excepto en metalenguaje); las preposiciones (puede ir una negación ante una preposición, pero afectará a todo el sintagma introducido por dicha preposición); los relativos y los adyacentes de sintagmas en forma de cláusula. Como se verá, el español de Centroamérica no diverge de esta caracterización.

Negación y foco

Así, cualquier elemento funcional puede recibir la negación, siempre que esté focalizado, por lo tanto la influencia del énfasis es

33 David Crystal, La muerte de las lenguas (Madrid: Cambridge University Press, 2000). 
decisiva. Para Sánchez ${ }^{34}$, el foco de la negación es aquel elemento sobre el que recae la exclusión o refutación, ejemplo (8a); y anota que generalmente la refutación está acompañada de una cláusula correctiva o sintagma que aporta la información que rectifica la del elemento negado. Existen varios modos de hacer dicha corrección: la conjunción sino (8b), una perífrasis de relativo (8c), o la estructura (y) no, (8d).

(8a) Carlos no perdió las llaves, sino que las olvidó en su casa.

(8b) No compró manzanas, sino papas.

(8c) No fue con Luis con quien llegó.

(8d) Fue con Luis, (y) no con Silvia.

La negación también puede ocupar un lugar característico ante el verbo o precede inmediatamente al foco; para ello, es indispensable un elemento correctivo introducido por sino que forme junto con el foco un solo constituyente inseparable, como en los ejemplos (9a) y (9b).

(9a) El gato cazó en el cafetal [no un ratón, sino un chapulín]. (9b) * El gato cazó [no un ratón] en el cafetal, [sino un chapulín].

\section{La negación en el español centroamericano}

A continuación se analiza la información correspondiente obtenida de la base de datos de PROLHISPA ${ }^{35}$. Ellos (cuadro 2) arrojan un total de 2476 instancias del operador $n o$, de las cuales 2380 , o sea el $96,2 \%$, se utilizó para negar el fv y solo 96 , el 3,8 \%, para el

34 Sánchez, 2577.

35 Es importante señalar que no se tuvieron en cuenta las frases en que únicamente aparecía el operador no o un TPN, como en las respuestas a las cláusulas interrogativas (Yes/No questions) para las cuales respondieron: ¡No! Además, la repetición seguida de un operador equivalió a uno solo; por ejemplo: ... pues, no no no poder dejar de beber ¿vaá? 
FN, lo cual corrobora la afirmación de Givón y Payne en cuanto a la preferencia de negar la cláusula en lugar de solo una parte de ella.

\section{Cuadro 2. Porcentaje de aparición del operador no ante fv o FN en el español centroamericano}

\begin{tabular}{|c|c|c|c|c|}
\hline \multicolumn{2}{|c|}{ fv } & \multicolumn{2}{c|}{ FN } & \multirow{2}{*}{ Total } \\
\hline $\mathbf{N}$ & \% & N & \% & 2476 \\
\hline 2380 & 96,2 & 96 & 3,8 & 246 \\
\hline
\end{tabular}

Negación de cláusula

Respecto a la negación de cláusula o de fv, que es la más frecuente, ofrecemos algunos ejemplos del español centroamericano, en (10), (11), (12), (13), (14) y (15).

(10) aaay, es que yo no tengo tiempo» (GT-H-1)

(11) eee cuando ella estaba no había la posibilidad de estudiar en una universidad eeee (HN-M-2)

(12) no le hagás caso» (ES-H-3)

(13) no me dejaba que yo fuera a jugar, si no estudiaba (NI-H-1).

(14) hay un puente pero no es peatonal (CR-H-2)

(15) uno no puede igualarse al cliente (PA-H-1)

Los anteriores ejemplos, y los porcentajes del Cuadro 2, demuestran que el español de Centroamérica no difiere del estándar, ni de la tendencia universal, al emplear generalmente la negación de cláusula y no la de constituyentes.

\section{Negación de constituyentes o de FN}

En cuanto a lo expuesto acerca de que cualquier elemento funcional puede recibir la negación, siempre que esté focalizado, noventa y seis $(3,8 \%)$ de las estructuras con el modulador no pertenecen 
al tipo de negación de constituyentes. A continuación se presentan ejemplos del operador no ante diferentes tipos de constituyentes.

- $\quad$ Ante SN / At

(16) a la hora que yo regreso todos están acostados o ya apunto de acostarse, entonces era como no tener tiempo para ellos (ES-M-1)

- $\quad$ Ante SN / OD

(17) Eso/entonces sí me gusta comprar, me gusta comprar, bueno, no ropa porque eso es más para las mujeres (HN-H-1)

\section{- $\quad$ Ante SN / Término}

(18) o sea, yo no creo en eso de no tener tiempo (GT-H-1)

\section{- Ante SADJ}

(19) fue una vida, pues, no no tan lujosa (NI-H-1)

\section{- Ante SADV}

(20) lo que usted les está explicando no memorísticamente (PA-M-3)

\section{- Ante SPREP}

(21) Y/pero uno iba a esasn... fiestas, pues a verse con su novio ¿veá?, no por otra cosa; (GT-M-3)

Del mismo modo, en el español centroamericano la exclusión o refutación recae en el foco de la negación y que algunas veces está acompañada por una cláusula correctiva o sintagma que aporta la información que rectifica la del elemento negado, con en los ejemplos (22) y (23). 
(22) Sí, claro, exacto. Eeee a tomar un café, bueno, no un café porque un café no me gusta en realidad, sino una granita ooo come/una granita ¿no sabe qué es? (HN-H-1)

(23) quiero un futuro, pero en realidad yo soy de la idea de no pensar mucho en eso, sino ir construyendo lo que hago ahorita, disfrutar lo que hago, con esto (GT-H-1)

\section{Negación y el orden de los constituyentes en español}

El único aspecto hallado en el corpus del español centroamericano que se aleja de lo aseverado tanto por Sánchez ${ }^{36}$ como por la $\mathrm{RAE}^{37}$ para el español, es el tipo de constituyentes que se puede encontrar entre el operador no y el verbo sin que se produzca una oración agramatical; suele señalarse como únicos elementos al reflexivo, al objeto directo y al indirecto; sin embargo, en los casos (6) repetido como (24), (7) repetido como (25), y (26), además del objeto indirecto, el intensificador mucho se halla entre el operador no y el verbo, sin que por ello se produzca una agramaticalidad para los hablantes, pues incluso uno de los informantes repitió la estructura en una misma frase. Todos los ejemplos emplean el verbo gustar y provienen de informantes de la capital guatemalteca y son utilizados por hombres $\left(1^{\circ}\right.$ y $2^{\circ}$ grupos etarios).

(24) Ajá, y los dulces típicos hay un montón, pero yo no/por ejemplo no como dulces típicos; no mucho me gustan (GT-H-1)

(25) a/en sí, así juegos no mucho les gusta; no, no les llama/así juegos comoo basquet o algo así nooo... deportes no les no les llama la atención (GT-H-2)

(26) ... a mí me gusta todo, de por sí lo que no mucho me gusta son esos nuevos grupos que han salido ahora ¿va? No mucho me gusta, como Link Biskit, Linking Park, no mucho me gustan, Gorilas y todo eso no mucho me gusta, pero sí una que otra me gusta (GT-H-2)

36 Sánchez.

37 Real Academia Española, Nueva gramática de la lengua española. Manual (Madrid: Espasa, 2010). 


\section{Conclusión}

El español centroamericano presenta una relativa homogeneidad en cuanto al uso de la partícula no como operador de negación, pues no existen mayores diferencias en cuanto a la tipología de la negación en el español empleado en las capitales centroamericanas, con excepción de los casos guatemaltecos en que el orden de los constituyentes se altera y que requieren particular atención. Este resultado contribuye a confirmar la premisa de PROLHISPA y el proyecto Variación tipológica en el español de Centroamérica, de la Universidad Nacional, que postula una homogeneidad formal en el español centroamericano y que las diferencias dialectales se hallan más en los componentes fonológico y léxico que en el morfosintáctico. El interés de este artículo, al analizar estructuralmente mecanismos de codificación (como la negación, entre otros) e identificar una correlación geográfica con el continum variacional surgido de dicho análisis fue precisamente puede establecer una correspondencia tipológica tomando al istmo centroamericano como una superficie.

\section{Lista de abreviaturas}

$\begin{array}{ll}\text { at } & \text { atributo } \\ \mathrm{CR} & \text { Costa Rica } \\ \mathrm{e} & \text { expresión } \\ \mathrm{ES} & \text { El Salvador } \\ \mathrm{fn} & \text { frase nominal } \\ \mathrm{fv} & \text { frase verbal } \\ \mathrm{GT} & \text { Guatemala } \\ \mathrm{H} & \text { hombre } \\ \mathrm{HN} & \text { Honduras } \\ \text { inf } & \text { infinitivo } \\ \mathrm{M} & \text { mujer } \\ \text { neg } & \text { negación }\end{array}$


NI

o

od

PA

OV

ovs

PROLHISPA

RAE

SADJ

SADV

SN

SV

SVO

TN

TPN

VO

VS
Nicaragua

operador

objeto directo

Panamá

objeto-verbo

objeto-verbo-sujeto

Programa de Lingüística Hispánica

Real Academia Española

sintagma adjetival

sintagma adverbial

sintagma nominal

sujeto-verbo

sujeto-verbo-objeto

término negativo

término de polaridad negativa

verbo-objeto

verbo-sujeto 